\title{
La machine admirable
}

Erhard Taverna

\section{«Dabei war alles überaus gut gemeint.»}

Es kommt selten vor, dass ein Personenname ohne weiteren Hinweis die Sache an sich bezeichnet. Erfinder, meist Techniker oder Ingenieure, werden damit geehrt. Etwas tragischer sieht es aus, wenn der Name eines Arztes für alle Zeiten zum Synonym eines Tötungsinstrumentes wird. Denn, anders als für Leute wie Colt und Kalaschnikow, bedeutet diese Ehrung für einen Mediziner mehr Bürde als Würde, ein Makel, der die Berufsethik unterläuft.

Dabei war alles überaus gut gemeint, als Joseph Ignace Guillotin (1738-1814), Dozent der Medizin in Paris, Advokat der Pressefreiheit und gewählter Depu-

tierter der Assemblée constituante, seine sechs Artikel zu einer Justizreform vortrug. Der zum Tod Verurteilte sollte, unabhängig vom Delikt, durch eine simple Mechanik geköpft werden. Das war 1789 durchaus ein Fortschritt, denn strafbare Mitbürger wurden je nach Verbrechen gevierteilt, gerädert, erhängt, verbrannt, verbrüht oder mit dem Schwert geköpft. Mit seinem Vorschlag befand sich der aufgeklärte Philanthrop in bester Gesellschaft. Bereits Diderot hatte in seiner Enzyklopädie viele gute Argumente für einen Vorschlag vorgebracht, der die Missetäter durch eine Vivisektion dem Fortschritt und damit der Gesellschaft dienstbar machen wollte. Auch Guillotin hatte ganz in der Denkweise damaliger Intellektueller vorgeschlagen, medizinische Experimente statt an Tieren an Verurteilten vorzunehmen. Der Mediziner, Vertreter des dritten Standes und Mitglied einer königlichen Kommission, suchte nach einer Lösung, die für alle Todesurteile eine möglichst schmerzlose Hinrichtung garantierte. Eine Maschine arbeitet zuverlässiger als ein Henker. Zudem war das Fallbeil in zahlreichen Ländern schon seit längerer Zeit im Gebrauch, dort allerdings den Adligen und Klerikern vorbehalten. Sein Vorschlag sollte das Strafverfahren vereinfachen und demokratisieren.

Sein Prestige überzeugte die Versammlung aber nicht. $\mathrm{Zu}$ ungeheuerlich war den Versammelten die Vorstellung dieser Maschine, zu enthusiastisch die Propagierung ihrer Vorzüge. Er wurde lächerlich gemacht und in der Presse mit ironischen Kommentaren überhäuft. Zwei Jahre später drohte die Republik unterzugehen. Im Inneren gab es Aufstände, an den
Grenzen siegte die Koalition der ausländischen Gegner. Die gleiche Assemblée verlangte nun von Guillotin eine neue Beurteilung. Doch dieser lehnte ab. Es war der Arzt Louis, Sekretär der chirurgischen Akademie und Leibarzt des Königs, der in seinem «Avis motivé sur le mode de décollation» eine verbesserte Version des schottischen Schafotts empfahl. Nach seinen technischen Plänen wurde der Apparat innerhalb einer Woche eingerichtet und darauf im Spital im Beisein von drei Ärzten, darunter auch Guillotin, an Leichen erprobt. Nach einigen Verbesserungen funktionierte die Maschine, und am 25. April 1792 fand die erste öffentliche Hinrichtung statt.

Das neue Gerät setzte sich durch, weil die Politiker, zusammen mit der zeitgleichen Erfindung des Telegrafen, dem Volk den sozialen und intellektuellen Fortschritt der Revolution demonstrieren konnten. Die Öffentlichkeit nannte das Fallbeil zuerst Louison oder Louisette, bis sich die Bezeichnung Guillotine durchsetzte. Unter Robespierre wurde sie zum

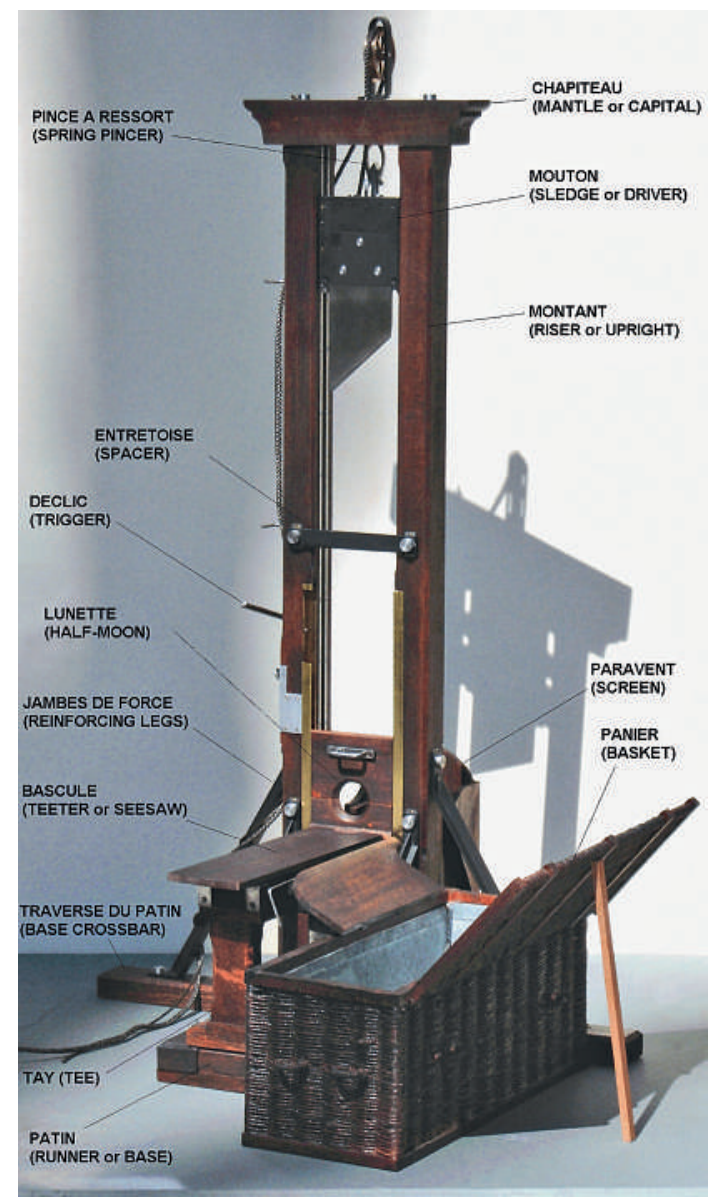




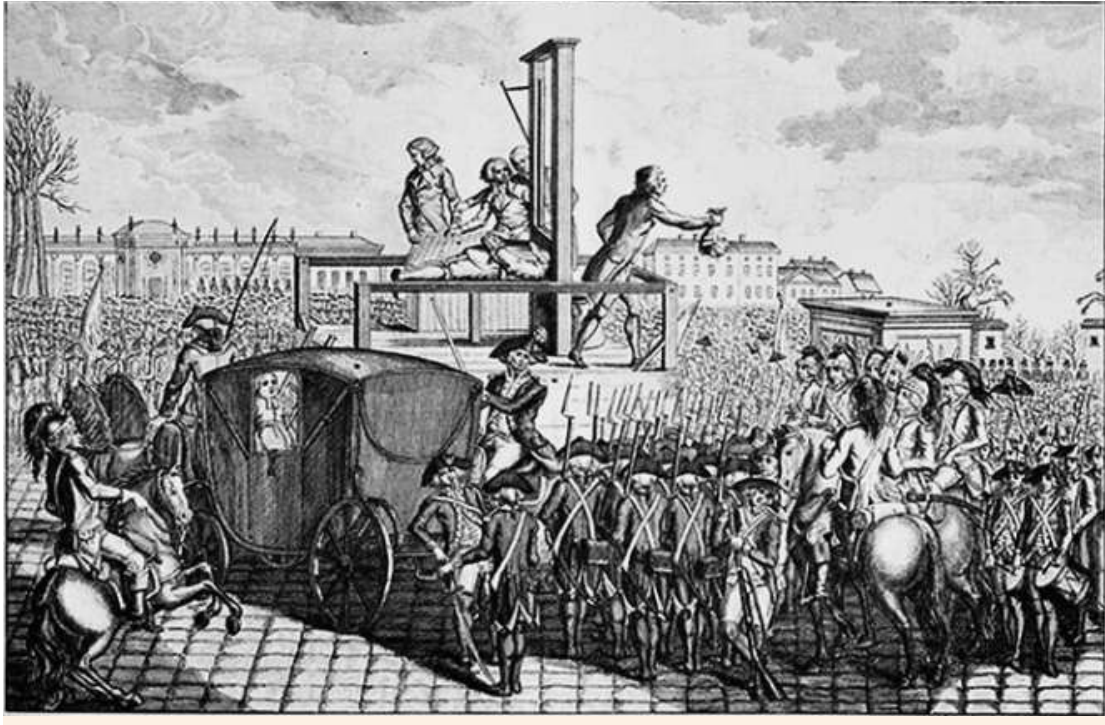

Während der Französischen Revolution herrschte Hochbetrieb an der Guillotine. Hier ein Kupferstich aus dem Jahr 1793, der die Hinrichtung Ludwigs des XVI. zeigt.

Gesetz, zu einem Symbol der neuen Herrschaft. Der Terror nahm seinen Lauf und kulminierte mit der Hinrichtung des Königs Ludwig XVI. am 21. Januar 1793.

Zahlreiche Akten belegen die politische Bedeutung der Guillotine. Die Schädelpyramiden wurden als Ausdruck des Volkswillens gefeiert, die Inszenierungen der «roten Messen» erzogen das Publikum im

\section{«Nach seinem Tod bat die}

Familie, die Bezeichnung der

Tötungsmaschine zu ändern.»

Sinne der «volonté générale», wie sie Robespierre gemäss Rousseaus Ideen verstand. Den Vollzug an einem absoluten Herrscher von Gottes Gnaden empfanden viele als einen Akt ähnlich dem Abendmahl, der die Legitimation des verurteilten Königs auf die Nation übertrug. Diese Definition der Volkssouveränität folgte exakt der monarchischen Logik: «L'Etat c'est moi.» Lieder und Litaneien be- zeugen, gemäss dem Historiker Arasse, die verbreitete Kanonisierung der Guillotine:

Sainte Guillotine, protectrice des patriotes, priez pour nous;

Sainte Guillotine, effroi des aristocrates, protégez-nous;

Machine aimable, ayez pitié de nous.

Machine admirable, ayez pitié de nous.

Sainte Guillotine, délivrez-nous de nos ennemis.

Mit dem schlechten Ruf dieses Danse macabre sollte Guillotins Name für immer verbunden bleiben. Er überlebte trotz eines kurzen Gefängnisaufenthalts die Massaker der Revolution und wurde 1800 zum Präsidenten der Gesellschaft gegen Kuhpocken ernannt. Napoleon Bonaparte und den Papst soll er vom Nutzen dieser englischen Erfindung überzeugt haben. Nach seinem Tod bat die Familie, die Bezeichnung der Tötungsmaschine zu ändern. Doch die Regierung lehnte den Antrag ab, worauf die Angehörigen sich neue Namen zulegten.

1830 wurde die Todesstrafe für Falschmünzerei, schweren Raub, Dokumentenfälschung, Planung eines Attentates, Brandstiftung und einige weitere Delikte abgeschafft. Die Zahl der Hinrichtungen ging stark zurück. Die schnelle Dekapitation führte unter Ärzten zu Diskussionen über den Zeitpunkt des Todes, denn viele Bürger waren überzeugt, dass die abgetrennten Köpfe noch für einige Zeit weiterlebten. Die Fotografien der abgeschlagenen Häupter begründeten Ende des 19. Jahrhunderts die moderne Anthropometrie und die Verbrechertypologie von Charcot, Lombroso, Galton und Betrillon. Schon zuvor war das Schafott mit der Staffelei eines Malers verglichen worden, der abgeschlagene Kopf wurde zum Porträt. So entwickelten sich aus den Regeln der Ästhetik die neuen medizinischen und polizeilichen Erkennungsmerkmale. In Frankreich wurde bis 1939 öffentlich guillotiniert, im September 1977 fand die letzte Hinrichtung statt, 1981 wurde die Todesstrafe abgeschafft.

\section{Literatur}

- Arasse D. La guillotine et l'imaginaire de la Terreur, Champs histoire, Paris: Flammarion;1987.

- Historia, Septembre 2011, № 777, Paris, www.historia.fr 